\title{
Aerodynamics of Boundary Layer Ingesting Fuselage Fans
}

\author{
Alejandro Castillo Pardo* \\ Whittle Laboratory \\ University of Cambridge \\ $1 \mathrm{JJ}$ Thomson Avenue \\ Cambridge CB3 ODY, UK \\ Email: ac2181@cam.ac.uk \\ Cesare A. Hall \\ Whittle Laboratory \\ University of Cambridge \\ $1 \mathrm{JJ}$ Thomson Avenue \\ Cambridge CB3 ODY, UK \\ Email: cah1003@cam.ac.uk
}

\begin{abstract}
Boundary Layer Ingestion (BLI) potentially offers significant reductions in fuel burn and pollutant emissions. The Propulsive Fuselage Concept features a fan at the back of the airframe that ingests the $360^{\circ}$ fuselage boundary layer. Consequently, the distortion at the fan face during cruise is close to radial. This paper aims to devise and test a fan design philosophy that is tuned to this inflow distortion.

Initially a free-vortex fan design matched to clean inflow is presented. The effects of $B L I$ on the aerodynamics of this fan are investigated. A series of design steps are then presented to develop the baseline fan into a new design matched to fuselage BLI inflow. Both fan designs have been tested within a low speed rig. The impact of the fan design changes on the aerodynamics and the performance with BLI are evaluated using the test results.

\footnotetext{
${ }^{*}$ Corresponding author.
} 
This paper presents the successful application of a unique experimental facility for the analysis of BLI fuselage fans. It shows that it is possible to design a fan that accepts the radial distortion caused by fuselage $B L I$ with a modified profile of work input. The new fan design was found to increase the work input by $4.9 \%$ and to improve the efficiency by $2.75 \%$ relative to a fan designed for clean flow. This new fan design has reduced loading near the hub to account for the incoming distortion, increased mid span loading and negative incidence towards the tip for tolerance to circumferential distortion off-design.

\section{INTRODUCTION}

Boundary Layer Ingestion (BLI) potentially offers significant reductions in fuel burn and pollutant emissions [1-3]. The BLI propulsor re-energizes the low-momentum fluid in the boundary layer, reducing the losses associated to kinetic energy diffusion and viscous dissipation in the wake [2]. The EU funded CENTRELINE project [4] aims to demonstrate the propulsive fuselage aircraft concept illustrated in Fig. 1. This configuration features an electrically driven fan at the aft of the airframe that ingests $360^{\circ}$ of the fuselage boundary layer. Consequently, the distortion at the fan face during cruise is essentially radial, whereas other Boundary Layer Ingestion (BLI) installations $[3,5]$ create a highly mixed circumferential and radial distortion. It should therefore be possible to minimise any losses in fan efficiency and stability margin due to BLI through a new design approach matched to the distorted inflow.

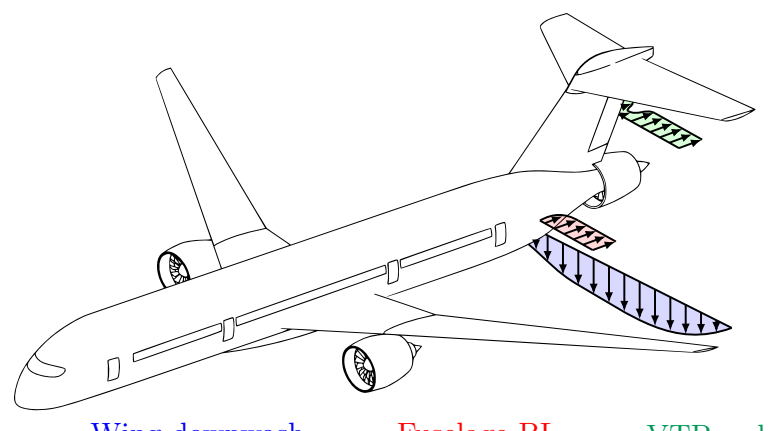

Fig. 1: Sketch of sources of distortion at the inlet of the fuselage fan. Adapted from Seitz et al. [4] 
There have been relatively few previous studies of the effects of radial distortion on fan aerodynamics and design. NASA tests in the 1970s with and without radial distortion [6-8] demonstrated how within a distorted region (an area of low total pressure) the rotor incidence angles are locally increased, loading up the blade sections and increasing the work input. It was noted how regions of high diffusion factor generally corresponded to high loss coefficient. The results also indicated how flow radially redistributes as a result of distortion, with mass flow migrating from regions of high total pressure towards low total pressure. This radial redistribution in mass flow was also described in detail in [9], for a mixed circumferential and radial distortion. In this case, the radial flow non-uniformity was reduced upstream and within a fan rotor. The redistribution was found to modify the rotor incidence and work variation.

The inflow of interest for a BLI fuselage fan at cruise is a severe and continuous hub-low radial distortion. This would normally tend to load up the inner sections of the rotor, leading to possible flow separation, combined with reduced loading towards the tip. This would be expected to reduce both fan efficiency and pressure ratio as shown in [6,7]. However, an improved radial loading distribution is possible if the rotor is carefully designed to accept the non-uniform inflow. At each spanwise location, the blades can be optimally aligned with the inflow and loaded such that they give the required work input whilst also remaining efficient. This paper aims to devise such a design philosophy for fuselage fans and to demonstrate it experimentally.

The paper starts by presenting the computational methods used for the fan aerodynamic designs and the experimental methods used for the measurements. In the following design section a conventional, free-vortex style fan design, Fan A, is presented that satisfies the non-dimensional work and flow requirements of the fuselage fan at cruise. Fan A has been tested in clean and distorted conditions representative of fuselage BLI. A new design, Fan B, is then presented that is improved from Fan A through a series of design steps for operation with fuselage BLI. Experimental measurements of fan B operating within a boundary layer have been taken. Comparisons of the numerical methods and the experimental results are presented to validate the design approach. The effects of radial distortion on the flow field are examined by comparing the measured results in clean and distorted flow. The main results section presents the measured changes in the 
aerodynamics and performance between Fan B and Fan A when operating within distorted flow.

This paper shows that it is possible to design a fan stage for fuselage BLI that gives improved efficiency and pressure rise relative to a conventional design. The measured results are encouraging as they demonstrate that a fuselage fan operating in distorted flow can deliver equal performance to a conventional fan design operating in clean flow. The paper should be of interest to researchers working in the field of $\mathrm{BLI}$ engines and fan-distortion interaction.

\section{EXPERIMENTAL AND COMPUTATIONAL METHODS}

\section{Experimental Methods}

The experimental rig used for this research is a low-speed single-stage fan, known as the BLI rig. This facility was originally developed by Gunn et al. [9-11] for the analysis of low hub-to-tip radii ratio $\mathrm{BLI}$ fan aerodynamics. It has been updated substantially for this research.

Figure 2 presents the meridional view of the updated BLI fan rig. The rig is equipped with a long intake duct which enables the free interaction of the incoming distorted flow with the rotor. The annulus geometry has been modified to match the hub-to-tip radii ratio of CENTRELINE's aft-section fan. Additionally, new rotor and OGV blades have been fitted into the rig. The lowspeed nature of the rig does not allow compressibility effects to be replicated. Nevertheless, velocity triangles representative of CENTRELINE's full-scale transonic aft-section fuselage fan are reproduced by matching the full-scale design flow coefficient and stage loading coefficient selected at aircraft level in [4] and presented in Table 1. Previous work performed in this experimental rig showed that compressible flow features and Reynolds number effects alter the details of the fandistortion interaction, but not the principles [9].

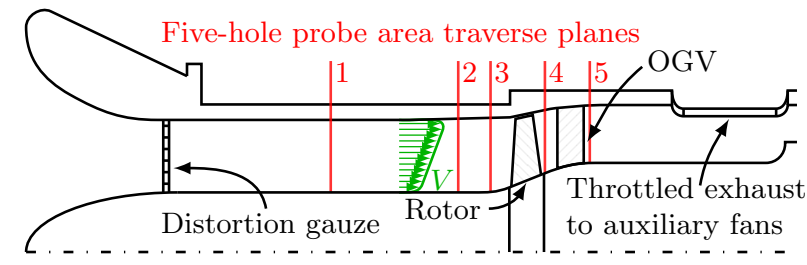

Fig. 2: Meridional view of the BLI fan rig indicating locations of measurement stations 
Table 1: Design point parameters for the BLI fan rig

$\begin{array}{lc}\text { Flow coefficient } \phi & 0.69 \\ \text { Stage loading coefficient } \Psi & 0.44 \\ \text { Rotor inlet tip Mach number } & 0.16 \\ \text { Rotor tip Reynolds number } & 2.1 \cdot 10^{5} \\ \text { Rotor hub-to-tip radii ratio } & 0.51 \\ \text { Running tip clearance (\% span) } & 0.35 \\ \text { Number of rotor, OGV blades } & 20,30\end{array}$

Detailed pressure measurements were carried out at five axial stations with a five-hole pneumatic probe area traverse system. The five-hole probe measured the time-average values of stagnation pressure, static pressure, swirl flow angle, and radial flow angle. Based on these properties and the incompressibility of the flow, the velocity field, work input, and loss sources were fully resolved. The probe was calibrated over a range of yaw and pitch angles between $+35^{\circ}$ and $+35^{\circ}$ using the procedure reported in [12]. The pressure probe was traversed radially and azimuthally across 12 degree sectors at stations 1-5. Measurements were taken with a resolution of 29 radial and 13 circumferential positions at stations 1-4. The circumferential resolution was doubled downstream of the OGV, at station 5, to capture the blade wakes in greater detail.

Experimental tests have been carried out for clean and distorted inlet boundary conditions. The distortion chosen for this study is an axisymmetric but radially non-uniform profile of axial velocity and therefore stagnation pressure. This profile represents the boundary layer velocity profile found at the inlet highlight plane of CENTRELINE's aft propulsor at the design flow coefficient $[4,13]$. Note that for this initial design phase of the project, the inflow is assumed to be purely axial and axisymmetric. Other sources of distortion have been neglected.

Flow conditioning gauzes, which are shown in Fig. 3, were installed at the intake of the rig to generate the target inlet velocity profiles for clean and BLI inflows. The gauzes, which comprise thousands of small blades with precisely controlled geometry, were designed using the method developed by Taylor [14] and 3D printed as a single thick sheet. This method makes use of 3D CFD simulations to design the vanes that form the gauze. The resultant inlet profiles have been 


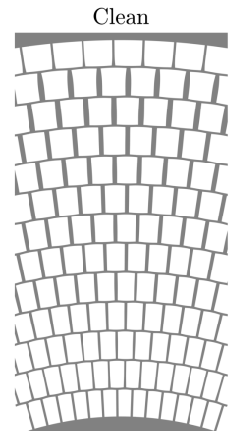

a)

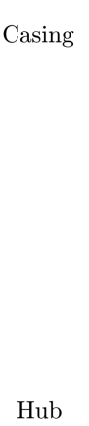

Hub

b)

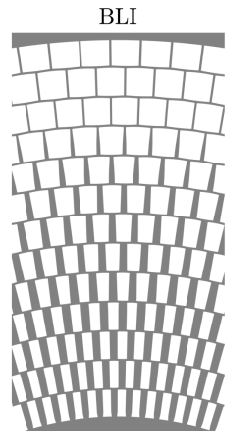

Fig. 3: Flow conditioning gauzes, a) Clean, b) BLI distortion

measured at station 1 to verify the effectiveness of the distortion gauzes.

Figure 4(a) presents the comparison between the target, numerical gauze results and the measured clean inlet boundary conditions. A nearly constant axial velocity has been successfully measured downstream of the gauze. The largest discrepancy between the target and the attained profile can be observed around $90 \%$ span. Marginal spatial noise caused by discrete vanes can be observed in the contour. Figure 4(b) presents the effectiveness of the gauze for the BLI inflow. Very good agreement between the experimental measurements and the target profile is observed with exception of the 10-30\% span region. In this section CFD simulations underestimated the pressure losses, leading to thicker blades with larger losses and consequently lower velocity.

\section{Computational Methods}

Single-passage, steady-state simulations were carried out using the GPU-accelerated CFD code Turbostream [15]. Turbostream is a 3D, unsteady, Reynolds-averaged Navier Stokes solver running on structured multi-block meshes. The one-equation Spalart-Allmaras turbulence model [16] was used for all simulations along with adaptive wall functions and a $y+$ value of approximately 5 on all solid walls.

Figure 5 shows the meridional view of the computational domain, which is an accurate representation the experimental setup of the $\mathrm{BLI}$ rig. The domain was extended 0.6 fan diameter upstream and 1 diameter downstream of the fan stage to allow enough space for the fan-distortion interaction to take place. The inlet coincides with measurement station 1 and the CFD outlet was 

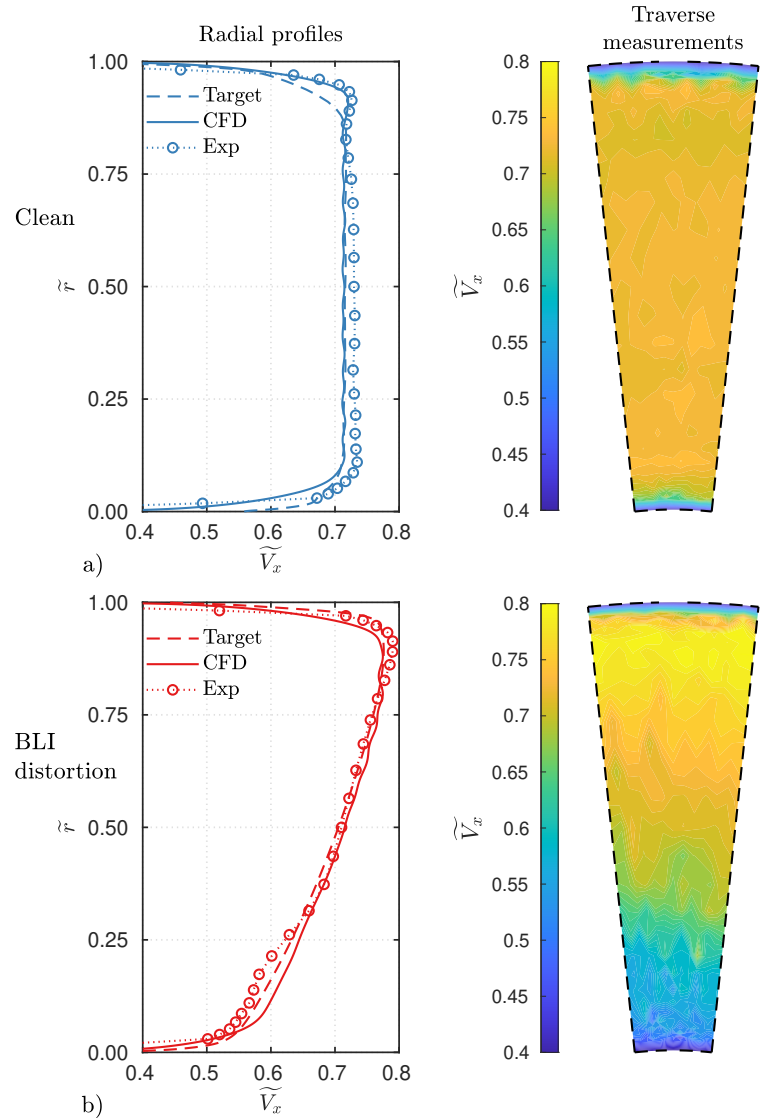

Fig. 4: Comparison of target, CFD and experimental measurements of axial velocity behind the conditioning gauzes at design point: a) Clean, b) BLI distortion

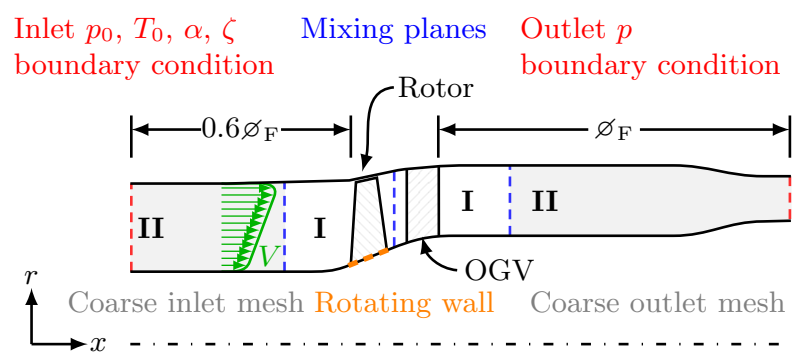

Fig. 5: Meridional view of the BLI fan computational domain showing the fine (I) and coarse (II) mesh regions

located at the rig exhaust. The structured multi-block computational mesh was generated using NUMECA IGG/Autogrid5 [17]. The computational domain was split in two fine mesh regions (I) and two coarse mesh regions (II). Rotor and OGV blades were contained within (I) subdomains, which were characterized by an $\mathrm{O} 4 \mathrm{H}$ topology with an $\mathrm{O}$-mesh around each blade. In contrast, 
a $\mathrm{H}$ topology was used to mesh the inlet and outlet subdomains (II). Around 2.5 and 1.9 million nodes were used per rotor and OGV blade passage, respectively. Previous studies showed the suitability of this setup for the aerodynamic analysis of distortion ingesting fans $[9,18]$.

Radial distributions of stagnation pressure and flow angles were prescribed as inlet boundary conditions of the numerical model. Two types of simulations were run: clean and distorted. For clean flow cases uniform inlet flow conditions were applied. Any non-uniformity of the flow was contained within the hub and casing boundary layers. For distorted cases axisymmetric, but radially non-uniform profiles of stagnation pressure matching the target fuselage boundary layer were applied at the inlet of the domain. The operating point of the model was controlled by varying the static pressure specified at the outlet boundary of the convergent nozzle.

\section{FUSELAGE FAN DESIGN}

This section firstly describes the baseline fan design, fan A. It then explores how the incidence and work input for this fan are modified with BLI. This leads to the steps taken to obtain a new fan design, fan B, adjusted for the inlet distortion shown in Fig. 4. Both fans have been designed for the target inlet profiles found in the full-scale configuration $[4,13]$ and shown in Fig. 4. Rotor and stator blades have been designed using five 2D sections (0, 25, 50, 75 and 100\% span), intermediate sections have been obtained using cubic spline interpolation. $1 \mathrm{~mm}$ radius fillets have been modelled to replicate the manufacturing limitations and no endwall contouring has been performed. Note that both fan designs $A$ and $B$ are matched to the overall design parameters detailed in Table 1. Both designs also use the same stator design, which was aligned to the exit flow angles from fan A operating in clean flow.

\section{Fan A: Conventional free-vortex design}

The rotor of fan A was designed to give a spanwise distribution of flow turning that produces approximately constant $\Delta h_{0} / U_{\text {mid }}^{2}$ as shown in Fig. 6(a) for clean conditions. The leading edge (LE) metal angle has been tailored to operate at minimum pressure loss incidence at the design point flow coefficient. An example of streamlines around the leading edge for minimum pressure loss incidence are shown in Fig. 7(b). The blade chord distribution was chosen to reduce Lieblein's 
diffusion factor DF [19] to a value under 0.35 as presented in Fig. 6(b) for uniform inflow. Note that endwall effects lead to larger values of loading and DF at the hub and casing.
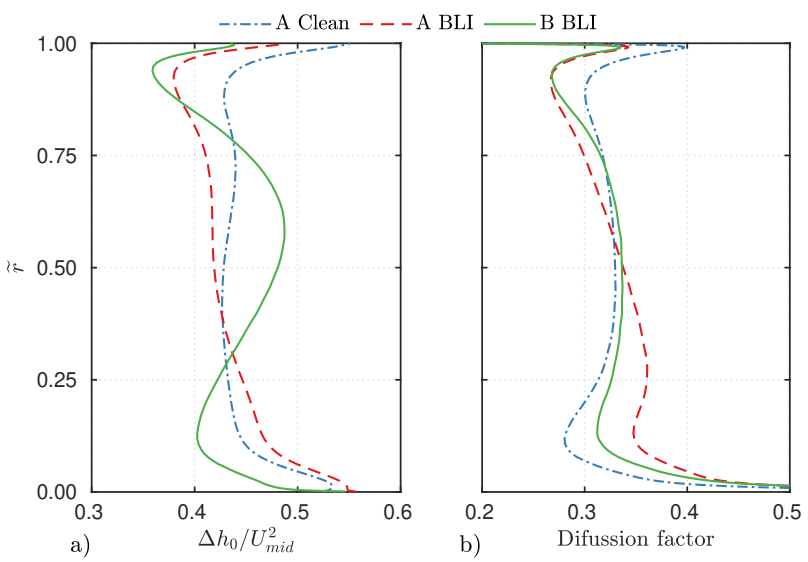

Fig. 6: Computed spanwise distributions of: a) work loading and b) Lieblein's diffusion factor

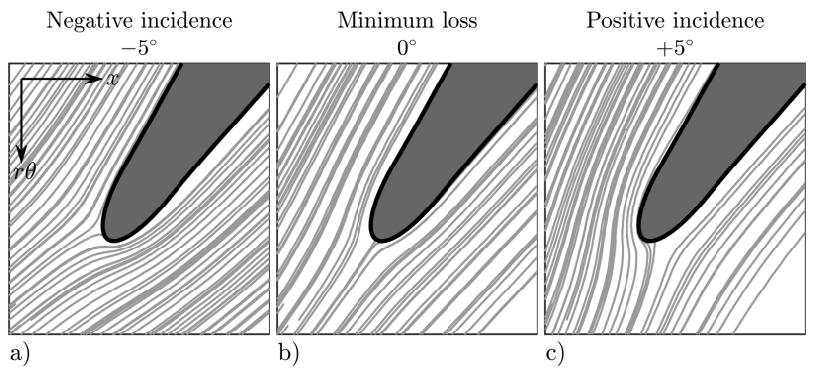

Fig. 7: Streamlines around the blade leading edge for fan A in BLI flow

The effects of BLI on incidence and work input

Figure 8 shows a velocity triangle sketch to illustrate the effects of flow coefficient changes due to fuselage BLI. When operating with distortion due to fuselage $\mathrm{BLI}$, inner sections of the fan blade are subjected to an axial velocity deficit. The midspan region operates at the design conditions and the outer sections are at increased axial velocities. Consequently, the region next to the hub operates at high incidence, while the tip sections of the blade operates at negative incidence. This leads to a movement of the leading edge stagnation point as observed in Fig. 7 for $\pm 5^{\circ}$ incidence. 
The effects of BLI on section incidence are further observed in Fig. 9, where predicted changes in the pressure distributions are presented. The midspan section of fan A operates at design incidence even with the ingestion of a boundary layer. The hub loading is increased with BLI and operates at positive incidence. The tip moves towards negative incidence and reduced loading with BLI.

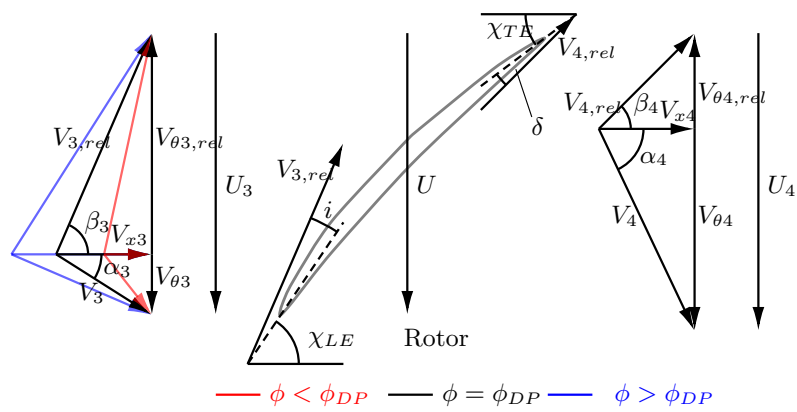

Fig. 8: Rotor blade velocity triangle changes due to boundary layer ingestion for fan A in BLI flow
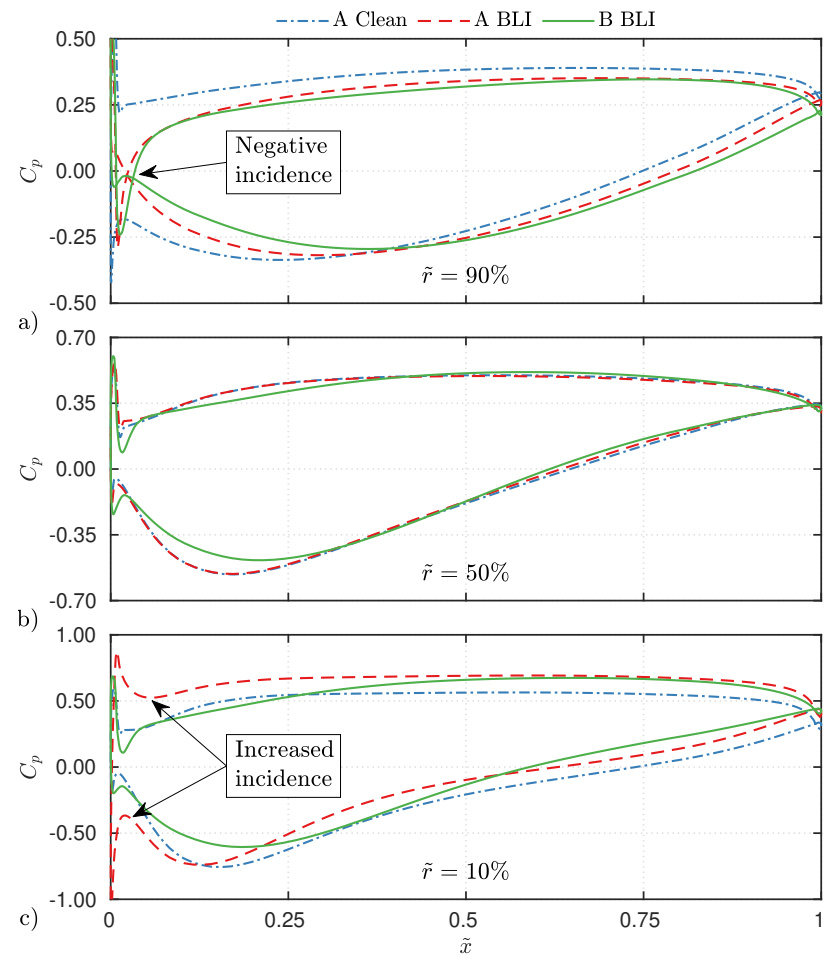

Fig. 9: Pressure coefficient distributions of fan A and fan B in clean and BLI conditions - a) $10 \%$ span, b) $50 \%$ span, c) $90 \%$ span 
To evaluate the effect of radial distortion on the local loading, the Euler work equation (Eq. 1) can be non-dimensionalized, as shown by Eq. 2.

$$
\Delta h_{0}=U_{4} V_{\theta 4}-U_{3} V_{\theta 3}
$$

$$
\frac{\Delta h_{0}}{U_{3}^{2}}=\frac{U_{4}^{2}}{U_{3}^{2}}+\frac{V_{x 3}}{U_{3}} \frac{U_{4}}{U_{3}} \frac{V_{x 4}}{V_{x 3}} \tan \beta_{4}-\frac{V_{x 3}}{U_{3}} \tan \alpha_{3}
$$

For this specific type of distortion, the dominant term affecting the local loading of the blade $\Delta h_{0} / U_{3}^{2}$ is the local flow coefficient $V_{x 3} / U_{3}$. The deficit of axial velocity at the inner sections results in increased local loading and diffusion factors (since $\beta_{4}<0$ ). In contrast, the tip sections get unloaded due to the excess of axial velocity. These trends are illustrated in Fig. 6 by the computed changes in work input for fan A due to BLI. The large extent of the unloaded region towards the tip leads to the significant overall loss in stage loading coefficient observed in Fig. 10(a). The off-design incidence results in a degradation of the isentropic efficiency of the rotor as presented in Fig. 10(b).

\section{Fan B: Design for BLI Distortion}

Step 1: Realignment of the Leading edge

Figure 9(a) and Fig. 9(b) show the pressure distribution of the $10 \%$ and $50 \%$ span section of fan $B$ realigned for minimum loss with BLI distortion, operating condition presented in Fig. 7(b). At $10 \%$ span, the positive incidence and section loading is significantly reduced. The incidence and loading have also been increased at midspan. Towards the tip, the blade has been restaggered to operate at negative incidence at design point (Fig. 12(a)).

Although the flow is assumed to be axisymmetric during the design phase, the fuselage fan 

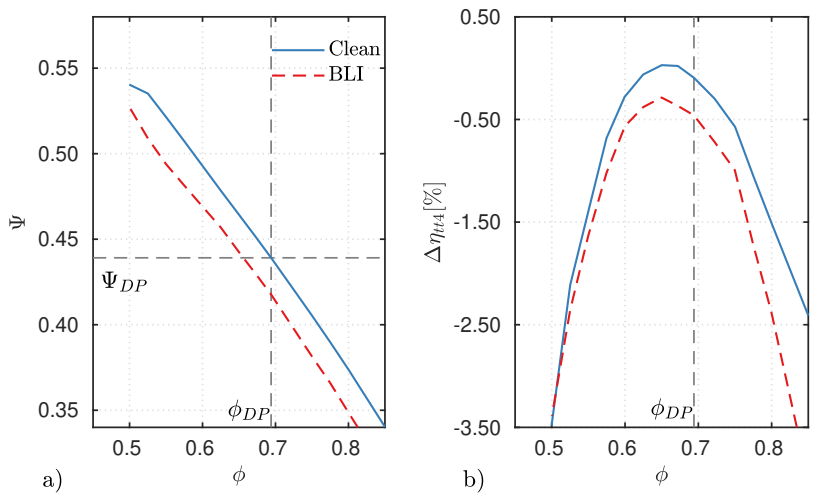

Fig. 10: Computed effect of distortion on fan A characteristics at constant rotor speed: a) stage loading, b) isentropic efficiency

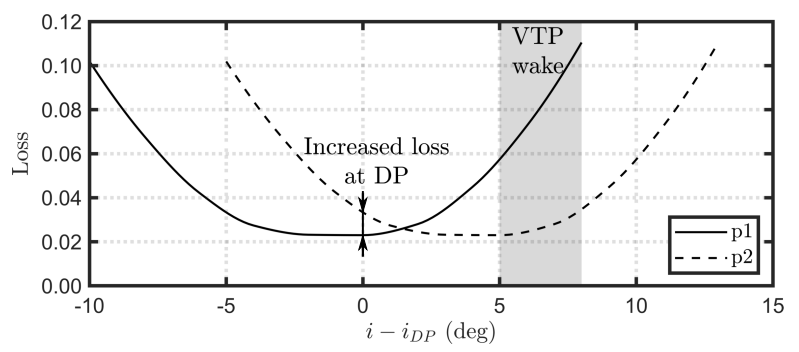

Fig. 11: Computed rotor tip sections loss loops

needs to be tolerant to large incidence fluctuations such as the ones caused by the vertical tail plane (VTP) wake, Fig. 1. Figure 11 presents the computed loss bucket curves for two rotor tip sections. When section $p 1$ operates at its design point incidence, the loss is minimum. For high values of negative or positive incidence the loss increases substantially. The shaded area illustrates the expected range of incidence found within a VTP wake. To increase the tolerance of the profile to such a distortion, section $p 2$ is shown, which is the same as $p 1$ but restaggered by $5^{\circ}$ to operate at negative incidence. This section is expected to operate within the VTP wake without high loss or instability at the expense of reduced efficiency at design point. This approach was applied progressively from $75 \%$ span to the tip, as shown in Fig. 12(a).

\section{Step 2: Midspan Blade Loading}

A radial distribution of work for fan $B$ was chosen that produced a midspan loaded blade, as shown in Fig. 6(a). As shown above, the rotor hub work tends to be increased by BLI, leading to 
high hub diffusion factors and high angles into the stator. The tip of the blade can be susceptible to incidence excursions and needs to have good operability. A mid loaded blade was therefore chosen as this was expected to provide the required work input with good efficiency and stability. Once the sections next to the tip were restaggered for negative incidence, the amount of turning was reduced further to improve the tolerance to circumferential distortion. The inner sections were unloaded until the diffusion factor was reduced to acceptable levels (see below).

\section{Step 3: Controlling the Diffusion Factor}

The restaggering and recambering steps described above lead to a blade with high values of diffusion factor at midspan. To reduce the diffusion to values below 0.35 , the solidity was progressively increased from the hub to $50 \%$ span, as shown in Fig 12(b) and progressively reduced to the original value at the tip. The final radial profiles of work and DF of fan B in BLI conditions are as presented earlier in Fig. 6.

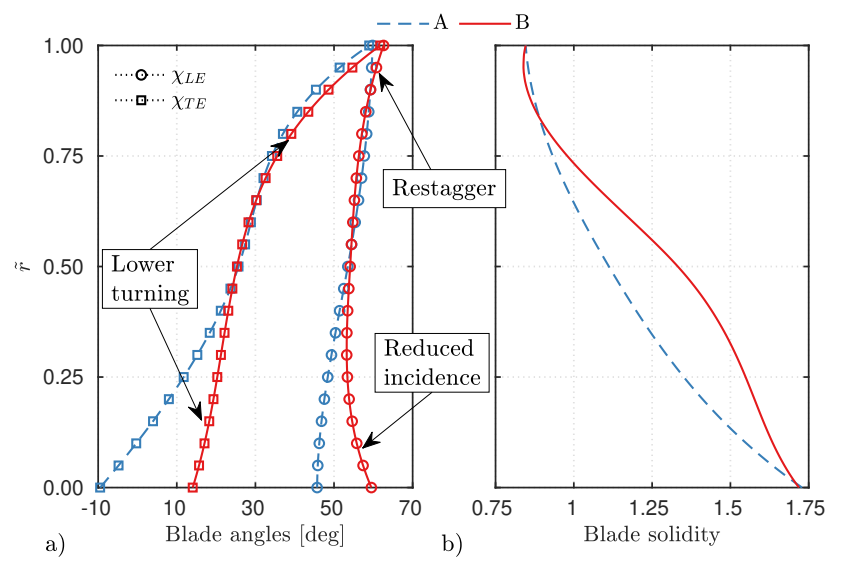

Fig. 12: Rotor blade geometry: a) Metal angles, b) Solidity

Three blade sections for both fan $A$ and fan $B$ are shown in Fig. 13. The larger $50 \%$ span chord shown for design B in Fig. 13 is consistent with the increase in solidity shown in Fig. 12(b). A 3D view of fan $A$ and fan $B$ are presented in Fig. 14. Note that there has not been any detailed mechanical analyses of fan A or B, but to reduce the unsteady bending moments of the blade, the sections have been stacked radially on their centroids. 


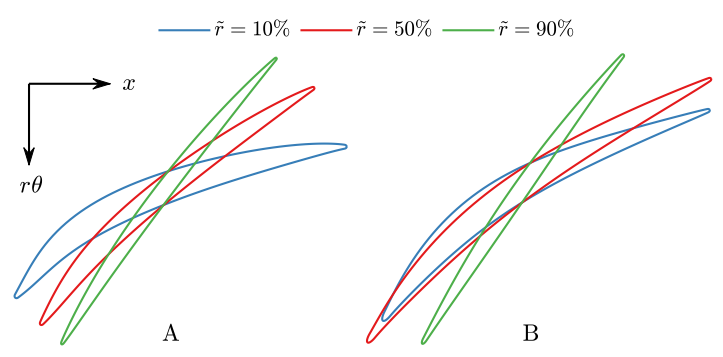

Fig. 13: Rotor blade sections at 10,50 and $90 \%$ span of fan $A$ and fan $B$

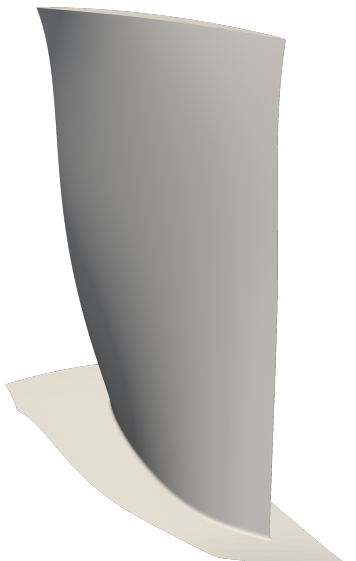

A

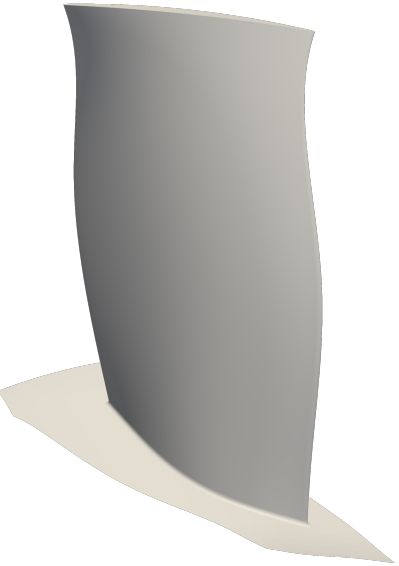

B

Fig. 14: 3D view of fans $A$ and $B$ rotor blades

\section{RESULTS}

\section{Flow field with fuselage BLI}

Figure 15 presents measured (symbols) and computed (lines) spanwise distributions at the fan face (s3), outlet of the rotor (s4) and downstream of the OGV (s5) for fan A. The distortion gauze installed at the inlet of the rig aims to replicate CENTRELINE's target velocity profile. As it was shown in Fig. 4, the underestimation of the vane pressure losses by the CFD code during the gauze design reduces the velocity attained between 10 and $30 \%$ span. The deficit of axial velocity propagates downstream and has been consistently measured at the fan face (Fig. 15(a)). The flow redistributes across the rotor, moving the location of maximum velocity to $70 \%$ span. Additional migration of flow towards lower parts of the span is identified. The flow further redistributes and becomes more homogeneous through the OGV. The mass flow redistribution is consistently captured with numerical and experimental techniques. 

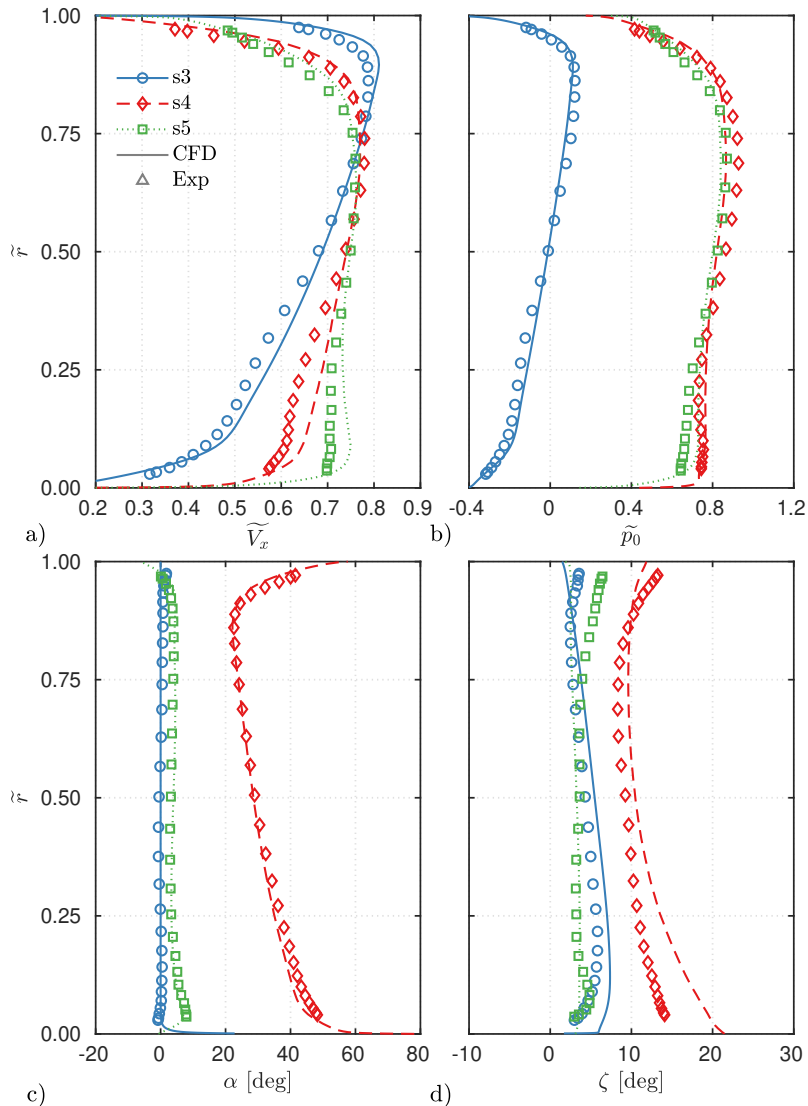

Fig. 15: Measured (symbols) and computed (lines) spanwise distributions of fan A - a) axial velocity, b) absolute swirl angle, c) stagnation pressure, c) radial angle

The small discrepancy in velocity measured between 10 and $30 \%$ span causes the rotor blade to operate at a higher incidence and work input than in the numerical simulations, increasing the turning and pressure rise. The associated increase in flow turning is observed in terms of absolute swirl angle at s4. There is very good agreement between experiments and CFD, with a marginal discrepancy on swirl angle. As a result, the stator blade is demanded to turn more the flow in the experiments, leading to increased total pressure losses downstream of the stage. In terms of radial angles, discrepancies of around $1^{\circ}$ have been found at mid-span, raising to up to $4^{\circ}$ near the hub.

The phenomena described above for fan A are observed in Fig. 16 for fan B. Very good agreement between measurements and computations of axial velocity is found except in the $10-30 \%$ span region upstream of the fan. The discrepancy is associated to the velocity profile generated 

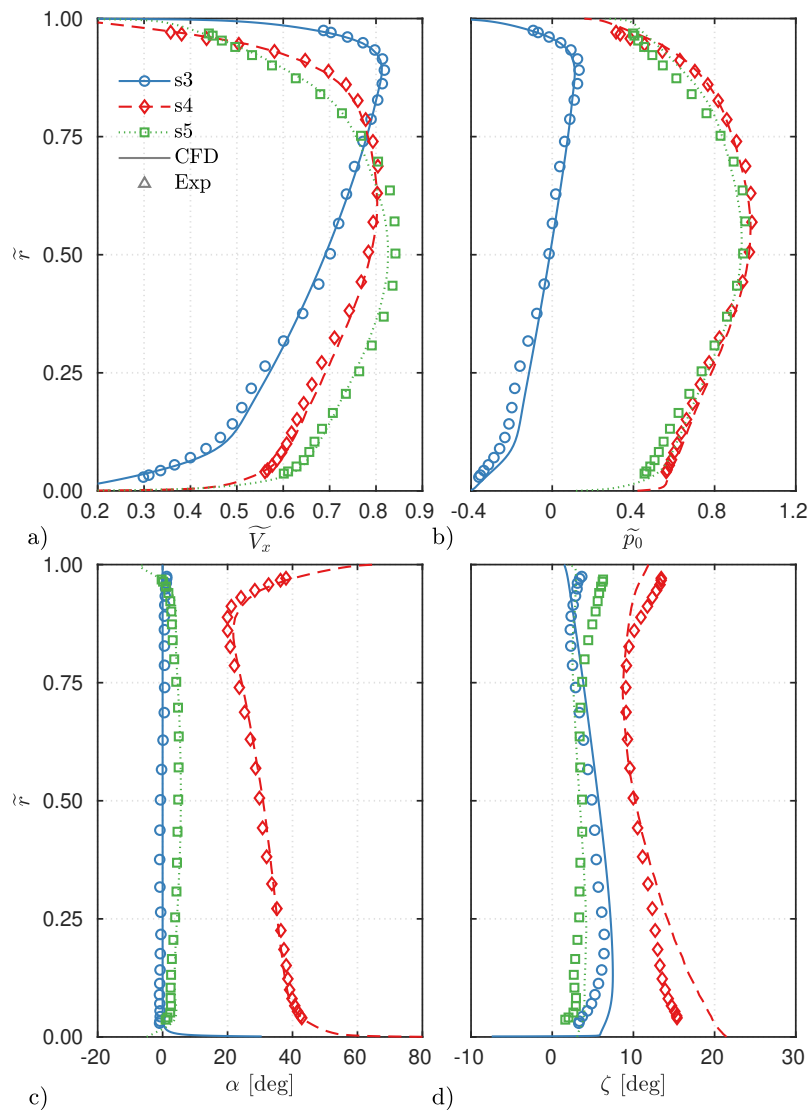

Fig. 16: Measured (symbols) and computed (lines) spanwise distributions of fan B - a) axial velocity, b) stagnation pressure, c) absolute swirl angle, c) radial angle

by the distortion gauze. A migration of the flow towards the mid span region is observed across the rotor and stator blades (Fig. 16(a)). Excellent agreement between computations and measurements is found for the swirl angle at the three stations of interest, which are translated into good agreement in stagnation pressure except for the deficit located at $10-30 \%$ span related to the conditioning gauze. A similar level of agreement is observed for the radial angles associated with fan B.

\section{Impact of BLI on Fan Aerodynamics}

Experiments with undistorted (clean) and distorted (BLI) inflows were performed with fan $\mathrm{A}$. To operate at the same flow coefficient in both types of inflow, the deficit of axial velocity observed below $50 \%$ span at the fan face (Fig. $17(\mathrm{a})$ ) is balanced by an excess of velocity above $50 \%$ span. 
The drop in radial angle at the fan face observed in Fig. 17(f) for the BLI case indicates a radial migration of flow towards the region of lower stagnation pressure upstream and through the rotor. Radial flow redistribution is further investigated in Fig. 18(a), where the change in normalized radius of particles seeded at a given radius $\widetilde{r_{1}}$ relative to the clean case is shown for different axial locations. Around a chord upstream of the rotor, the flow starts to redistribute towards lower span regions. By the time the air leaves the rotor most of the redistribution has taken place. A maximum level of $2 \%$ span hubwards redistribution relative to clean is found for particles seeded at $\widetilde{r_{1}} \approx 0.5$. Although of smaller magnitude, further redistribution takes place across the OGV leading to maximum values of around $2.4 \%$ towards the hub.

Recalling the velocity triangles presented in Fig. 8, regions of low and high axial velocity are linked with areas of increased and reduced relative swirl angle respectively. This is illustrated in Fig. 17(c), where the changes in relative swirl angle at s3 are shown along with the respective LE metal angle. Therefore, the blade continuously operates at off-design incidence inside the fuselage boundary layer. The computed $C_{p}$ distributions shown in Fig. 9 reaffirms the increased incidence found near the hub and the movement towards negative incidence near the tip.

In addition to variations in incidence, the amount of turning developed by the rotor blade changes as a results of BLI. Increased turning is observed in Fig. 17(d) below $50 \%$ span. The trend reverses towards the tip of the blade. Consequence of the modification of the swirl angle at s4 is the off-design incidence of the stator LE. Moreover, extra turning is required from the inner sections of the OGV, leading to higher pressure losses (Fig. 19).

Absolute swirl, axial velocity, and work input can be related using the Euler equation (Eq. 1). Assuming axial inlet velocity, it can be rewritten as $\Delta h_{0} / U_{m i d}^{2} \approx \widetilde{r_{4} V_{\theta 4}}=\left(U_{4} / U_{\text {mid }}\right) \widetilde{V_{x 4}} \sin \alpha_{4}$. Two distinct regions can be distinguished in Fig. 17(e). Below 35\% span the increase in swirl angle dominates over the deficit in axial velocity loading up the blade. On the upper part of the blade, the reduction in swirl angle outbalances the excess of axial velocity lowering the work input.

Blade loading is directly linked to the pressure rise generated by the blade. The increase in load in the inner sections (0-35\% span) results in a larger rise in stagnation pressure. Such an increase in pressure tends to balance the deficit of stagnation pressure due to the incoming inflow 

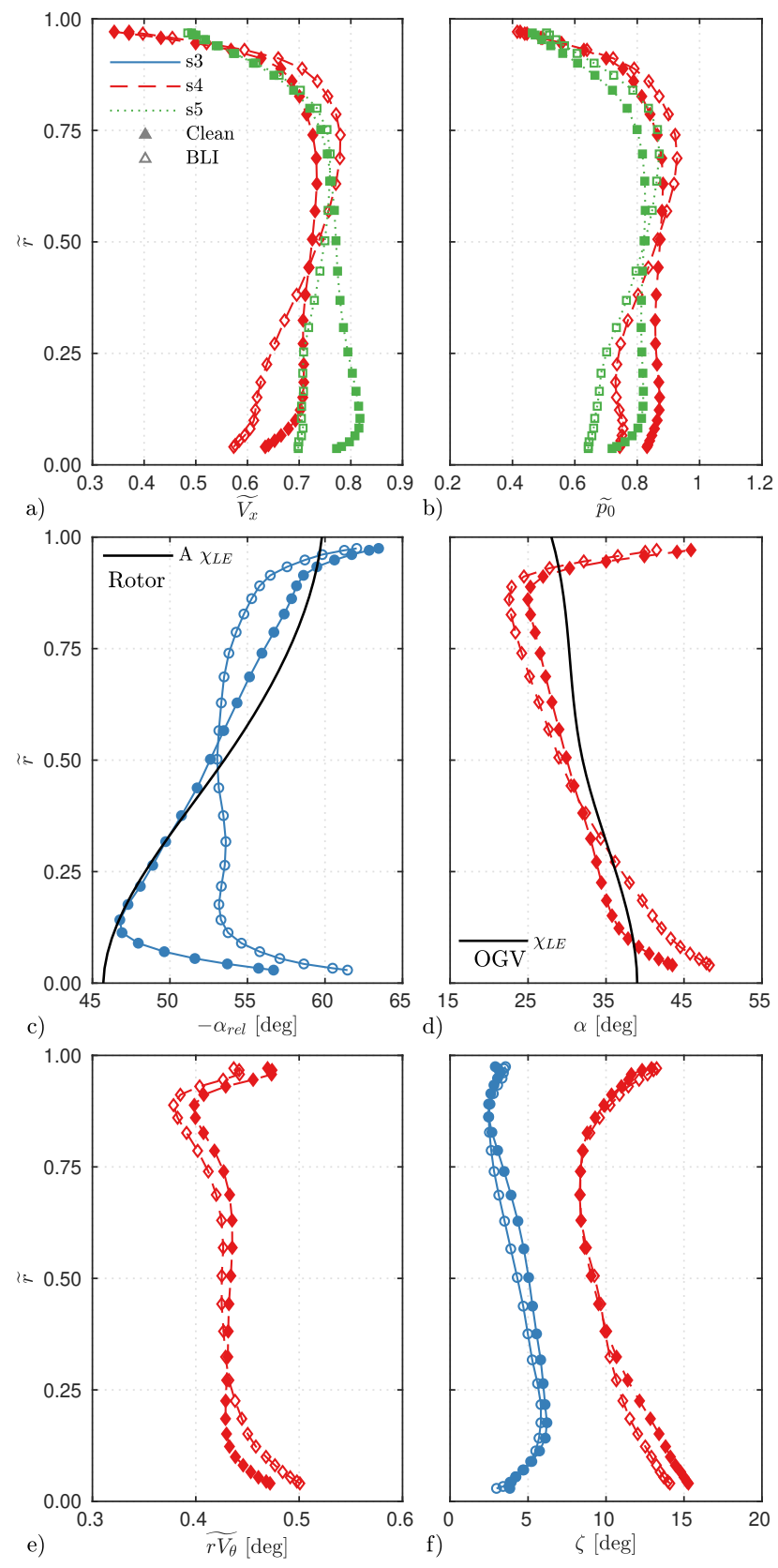

Fig. 17: Measured spanwise distributions for fan A in clean and BLI flows- a) axial velocity, b) stagnation pressure, c) relative swirl angle, d) absolute swirl angle, e) specific angular momentum, f) radial angle

as shown in Fig. 17(b) at s4. The rest of the rotor blade is unloaded, lowering the pressure rise. A large loss in stagnation pressure at $s 5$ near the stator hub is observed in Fig. 17(b), this is attributed to the larger size of the OGV hub corner separation presented in Fig. 19(b). 


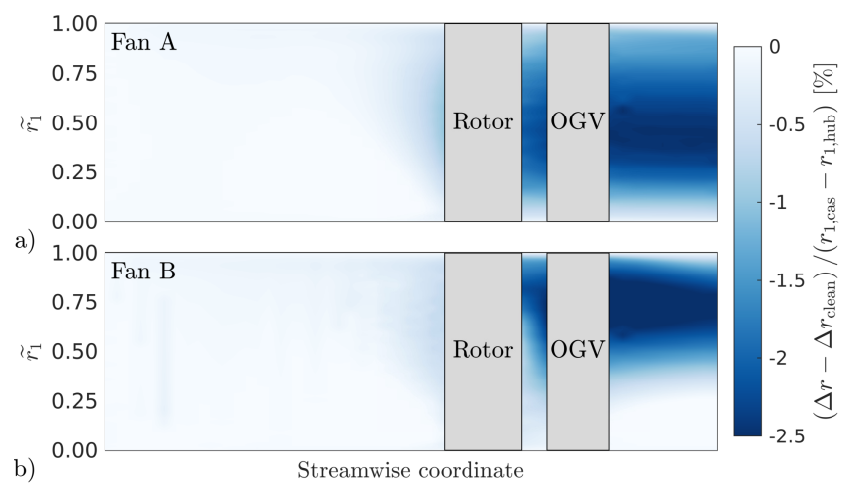

Fig. 18: Computed change in streamtube radius from station 1 relative to clean

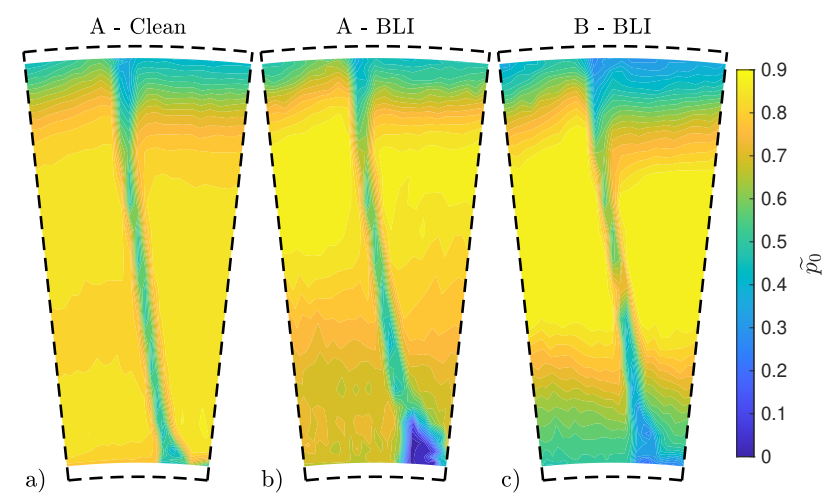

Fig. 19: Measured contours of stagnation pressure downstream of the OGV - a) fan A Clean, b) fan A BLI, c) fan B BLI

The flow phenomena presented in the previous paragraphs lead to changes in the global performance characteristics of fan A. The reduction in blade loading observed across most of the span results in a drop in stage loading coefficient $\Psi$, as shown in Table 2 for the design operating point $\phi=0.69$. The continuous operation of the blade at off-design incidence deteriorates the total-to-total isentropic efficiency $\eta_{t t 4}$. The combination of reduced loading and efficiency results results in a drop in rotor pressure rise coefficient $\psi_{t t 4}$.

Detailed area traverse measurements have been used to further quantified the effect of BLI on the performance of fan A at different operating points. The full characteristic line is presented in Fig. 20. A consistent drop in stage loading is observed for the complete operating envelope. In addition to the drop in loading, at least part of the blade is continuously operating at off-design incidence leading to a reduction in rotor isentropic efficiency as shown in $20(\mathrm{~b})$. It is noted that 
Table 2: Experimental measurements of the aerodynamic performance of fan $A$ and $B$ at design point

$\begin{array}{lccc} & \text { A Clean } & \text { A BLI } & \text { B BLI } \\ \phi & 0.69 & 0.69 & 0.69 \\ \Psi & 0.43 & 0.41 & 0.43 \\ \psi_{t t 4} & 0.85 & 0.81 & 0.86 \\ \eta_{t t 4}-\eta_{t t 4, \text { clean }}[\%] & - & -2.18 & +0.57\end{array}$
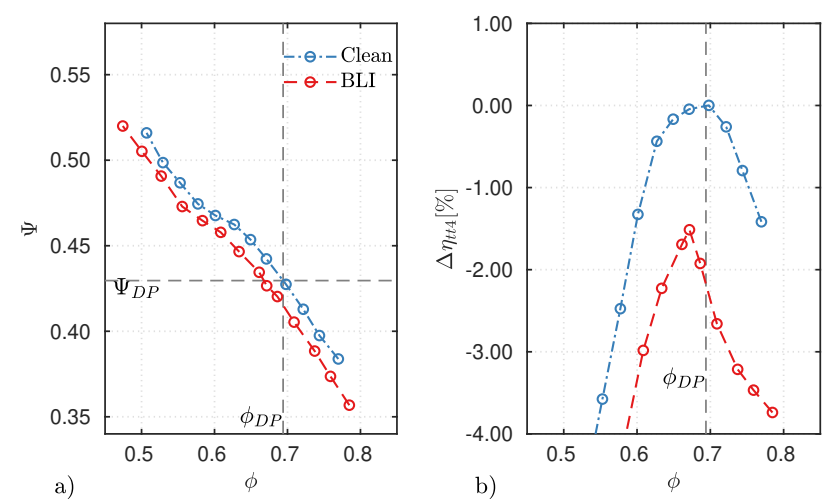

Fig. 20: Effect of distortion ingestion on the measured fan A characteristics at constant rotor speed: a) stage loading, b) isentropic efficiency

the flattening of the stage loading characteristic observed at $\phi \approx 0.55-0.6$ has been consistently measured in every configuration. This is related to the growth of the casing boundary layer on its diffusing section.

\section{Experimental Comparison of Fans A and B}

Radial flow migration for fans A and B are compared in Fig. 21(a) for fan B in terms of axial velocity. Fan $B$ tends to redistribute flow towards the mid-span region with higher intensity than fan A, which tends to move the flow hubwards to increase the uniformity of the flow. The difference in redistribution characteristics is more marked behind the OGV where a more uniform mass flow distribution is identified for fan A, compared with the highly mid-span concentrated mass flow for fan B. This is confirmed in Fig. 21(f) by the lower radial angles associated with fan A at s3 and s4. Figure 18(b) corroborates the trends identified in the experiments. Particles entering the stage at 

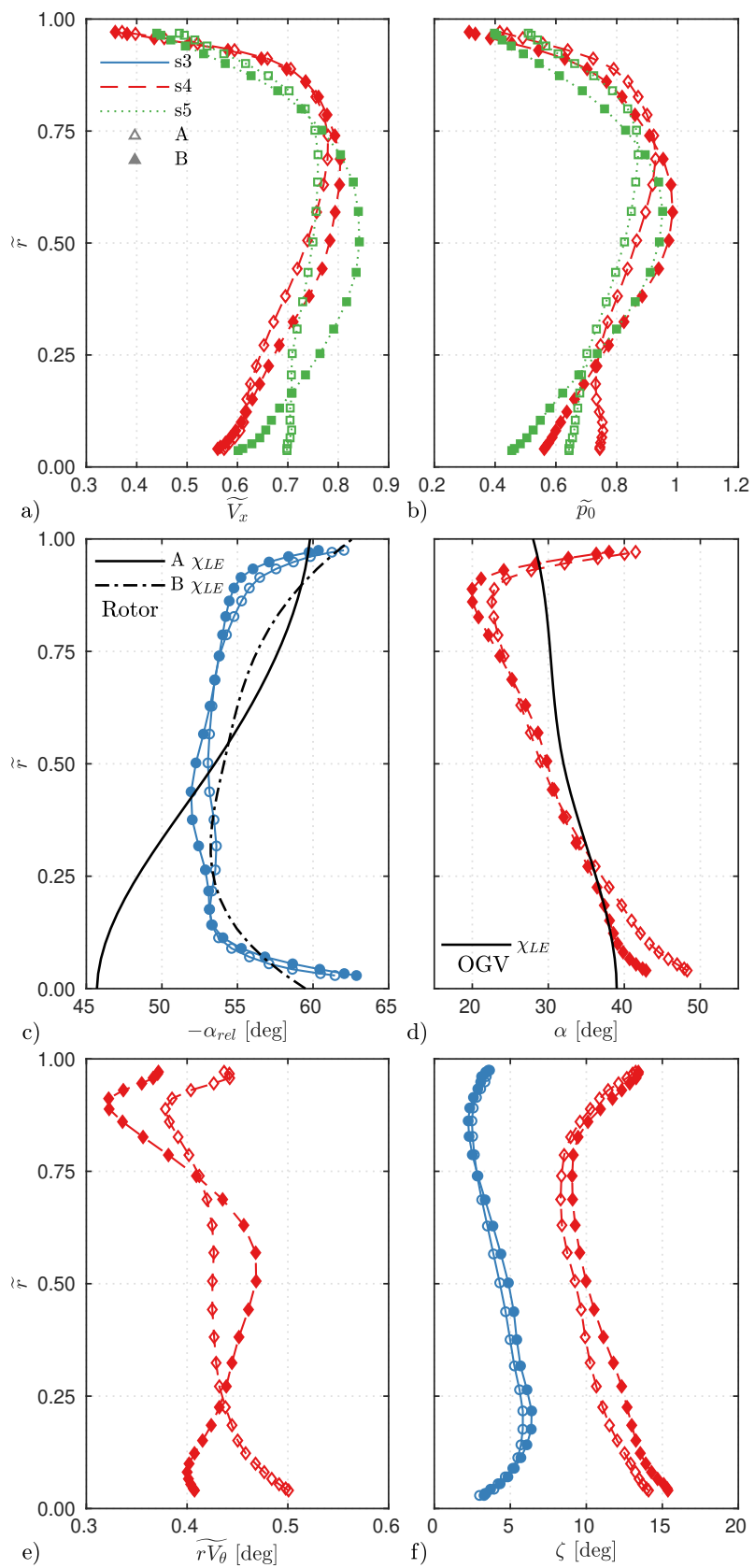

Fig. 21: Measured spanwise distributions for fans A and B in BLI flow- a) axial velocity, b) stagnation pressure, c) relative swirl angle, d) absolute swirl angle, e) specific angular momentum, f) radial angle

around $60-85 \%$ have migrated over $2.5 \%$ hubwards. In contrast particles seeded under $50 \%$ have barely moved towards the hub.

Small differences in relative swirl angle at the fan face are observed between fan geometries CASTILLO PARDO 
in Fig. 21(c). This can be attributed to a marginal offset in operating point and its effects of the performance of the distortion gauze. The realignment of the LE of fan B for optimum incidence can be observed in the plot. Additionally, the attempt to generate negative incidence near the tip is shown.

Following the realignment of the LE, the blade was recambered to deliver the desired work input. The amount of turning carried out by fan B is slightly increased between 20 and $75 \%$ span and decreased at the hub and tip (Fig. 21(d)). The extra turning in fan B could be achieved with improved efficiency using an OGV redesign. At the same time the hub and casing section of the OGV are required to turn the flow less, reducing the 3D secondary flows next to the walls.

A large increase in specific angular momentum is observed in Fig. 21(e) between 20 and 75\% span. This demonstrates that the midspan loaded design intent has been successfully achieved. Near the tip, the combination of reduced axial velocity and swirl angle results in low loading.
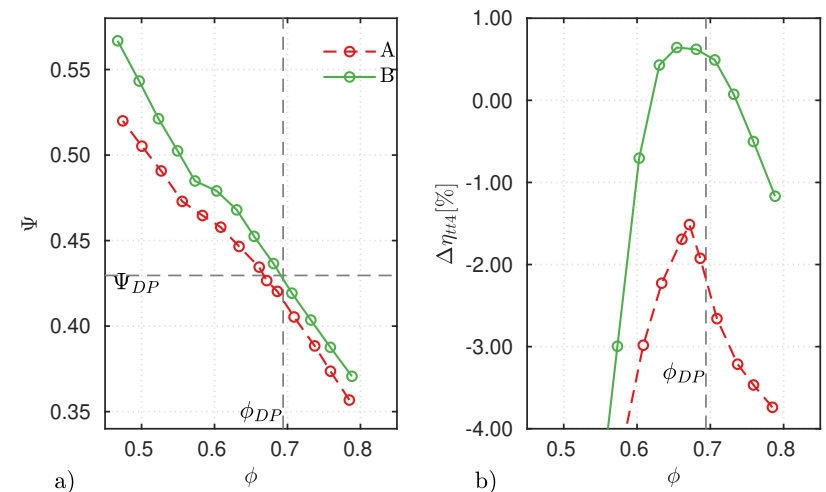

Fig. 22: Comparison of measured fans A and B distorted characteristics at constant rotor speed. a) stage loading, b) isentropic efficiency

The spanwise loading relates to the stagnation pressure rise distribution of rotor blade. Fan $B$ is able to generate a much larger pressure rise at mid-span than fan $A$, whilst maintaining lower values next to the hub and tip (Fig. 21(b)). A reduction in the OGV stagnation pressure losses near the hub can be identified as well in Fig. 21(b) for fan B. This can be associated with flow redistribution which takes place across the stator for fan $B$, easing the operation of the hub sections of the stator blade. This is confirmed in Fig. 19 by the smaller size of the hub corner 
separations downstream of the OGV in fan B.

Table 2 shows that fan $B$ is able to recover the performance lost by fan $A$ due to $B L I$ at the design operating point. The work transferred to the flow $(\Psi)$ is the same for fan B with BLI as it is for fan $A$ in clean flow. The rotor pressure rise for fan $B$ with $B L I$ is significantly higher than for fan $A$ with $B L I$ and marginally higher than fan $A$ in clean flow. This is linked to a small in rotor isentropic efficiency measured for fan B. The performance of fans A and B for BLI inflows at all operating points is presented in Fig. 22. Fan B is consistently able to transfer more work into the flow, reaching the design point requirements. Additionally, the total-to-total isentropic efficiency of design $B$ has been raised by over $2 \%$ across the full operating envelope.

\section{CONCLUSIONS}

1. A low-speed fan rig for the experimental testing of BLI fuselage fans has been successfully demonstrated for two fan designs.

2. A conventional free-vortex fan design has been designed, manufactured, and tested for clean and BLI inflow conditions.

3. The effect of the ingestion of radial BLI distortion on the free-vortex fan has been analyzed:

(a) Redistribution of the flow towards the low total pressure region near the hub is observed.

(b) The inner blade sections operate at high incidence and increased work load whereas the tip produces reduced work with negative incidence.

(c) The overall effect of BLI is the reduction in work input and efficiency.

4. A fan design optimized for aft-section fuselage BLI has been produced by:

(a) Realigning the leading edge metal angle to the inflow.

(b) Loading the midspan whilst unloading the hub and tip sections.

(c) Controlling the diffusion factor through custom work and chord distribution.

(d) Increasing the operating range of the tip sections.

5. The new design matches non-uniformity of the flow. It gives improved flow though the hub and increases the work input in the midspan blade sections, increasing the operating range.

6. The new fan design operating in $\mathrm{BLI}$ distortion increases the stage loading from 0.41 to 0.43 
and increases the efficiency by $2.75 \%$ relative to the conventional free-vortex design.

\section{ACKNOWLEDGMENTS}

This research is part of the CENTRELINE project, which has received funding from the European Union's Horizon 2020 research and innovation programme under Grant Agreement No. 723242. The authors are grateful to Turbostream for the use of their solver. At the Whittle Laboratory, Phoenix Tse and James Taylor are thanked for their technical advice, comments, and suggestions. Elliot Reed and Josh Firman are also thanked for their technical support manufacturing and assembling the rig.

\section{NOMENCLATURE}

\section{ROMAN SYMBOLS}

$C_{p}$ Pressure coefficient

$h$ Enthalpy

$i$ Incidence angle

$p$ Pressure

$r$ Radius, Radial coordinate

$T$ Temperature

$U$ Rotor blade speed

V Velocity

\section{GREEK SYMBOLS}

$\alpha$ Swirl flow angle

$\zeta$ Radial flow angle

$\theta$ Circumferential coordinate

$\rho$ Density

$\chi \quad$ Blade metal angle

CASTILLO PARDO 


\section{NON-DIMENSIONAL GROUPS}

$\widetilde{p} \quad$ Static pressure $=2\left(p-p_{01, \text { mid }}\right) / \rho U_{\text {mid }}^{2}$

$\widetilde{p_{0}} \quad$ Stagnation pressure $=2\left(p_{0}-p_{01, \text { mid }}\right) / \rho U_{\text {mid }}^{2}$

$\widetilde{r}$ Span fraction $=\left(r-r_{\text {hub }}\right) /\left(r_{\text {cas }}-r_{\text {hub }}\right)$

$\widetilde{r V_{\theta}} \quad$ Specific angular momentum $=r V_{\theta} / r_{\text {mid }} U_{\text {mid }}$

$\widetilde{V_{x}} \quad$ Axial velocity $=V_{x} / U_{\text {mid }}$

$\eta_{t t 4}$ Rotor total to total isentropic efficiency $\approx\left(\overline{p_{04}}-\overline{p_{03}}\right) / \rho U_{\text {mid }}^{2}\left(\overline{r_{4} V_{\theta 4}}-\overline{r_{3} V_{\theta 3}}\right)$

$\phi \quad$ Flow coefficient $=\dot{m} / \rho A U_{\text {mid }}$

$\psi_{t t 4}$ Rotor total to total pressure rise coefficient $=2\left(\overline{p_{04}}-\overline{p_{03}}\right) / \rho U_{\text {mid }}^{2}$

$\Psi \quad$ Stage loading coefficient $=\left(\overline{h_{04}}-\overline{h_{03}}\right) / U_{\text {mid }}^{2} \approx \overline{r_{4} V_{\theta 4}}-\overline{r_{3} V_{\theta 3}}$

\section{SUBSCRIPTS}

0 Stagnation quantity

1 Value just downstream of gauze

3 Value at rotor inlet

4 Value at rotor outlet

5 Value at OGV outlet

cas Value at the casing

hub Value at the hub

mid Value at midspan

rel Relative reference frame quantity

$x \quad$ Axial component

$\theta \quad$ Circumferential component

\section{ACRONYMS}

BLI Boundary layer ingesting

CFD Computational fluid dynamics

DF Lieblein diffusion factor 
Exp Experiment

LE Leading edge

OGV Outlet guide vane

TE Trailing edge

VTP Vertical tail plane

\section{REFERENCES}

[1] Smith, L. H., 1993. "Wake ingestion propulsion benefit". Journal of Propulsion and Power, 9(1), pp. 74-82.

[2] Drela, M., 2009. "Power balance in aerodynamic flows". AIAA journal, 47(7), pp. 1761-1771.

[3] Hall, C. A., Schwartz, E., and Hileman, J. I., 2009. "Assessment of technologies for the silent aircraft initiative". Journal of Propulsion and Power, 25(6), pp. 1153-1162.

[4] Seitz, A., Peter, F., Bijewitz, J., Habermann, A., Goraj, Z., Kowalski, M., Castillo Pardo, A., Hall, C. A., Meller, F., Merkler, R., Petit, O., Samuelsson, S., Della Corte, B., van Sluis, M., Wortmann, G., and Dietz, M., 2018. "Concept validation study for fuselage wake filling propulsion integration". In 31st Congress of the International Council of the Aeronautical Sciences (Belo Horizonte, Brazil, 9-14 Sept. 2018), International Council of Aeronautical Sciences.

[5] Uranga, A., Drela, M., Greitzer, E., Titchener, N., Lieu, M., Siu, N., Huang, A., Gatlin, G. M., and Hannon, J., 2014. "Preliminary experimental assessment of the boundary layer ingestion benefit for the d8 aircraft". In Proceedings of the 52nd AIAA Aerospace Sciences Meeting (National Harbor, Maryland, USA, 13-17 Jan. 2014), American Institute of Aeronautics and Astronautics, p. 0906.

[6] Sandercock, D. M., and Sanger, N. L., 1974. Some observations of the effects of radial distortions on performance of a transonic rotating blade row. Technical Note NASA TN D7824, National Aeronautics and Space Adminisitration, Dec.

[7] Schmidt, J. F., and Ruggeri, R. S., 1978. Performance with and without inlet radial distortion of a transonic fan stage designed for reduced loading in the tip region. Technical Paper NASA TP 1294, National Aeronautics and Space Administration, Aug. 
[8] Sanger, N. L., 1979. Effect of rotor meridional velocity ratio on response to inlet radial and circumferential distortion. Technical Paper NASA TP 1278, National Aeronautics and Space Administration, July.

[9] Gunn, E. J., and Hall, C. A., 2014. "Aerodynamics of boundary layer ingesting fans". In Proceedings of the ASME Turbo Expo 2014 (Düsseldorfd, Germany, 16-20 Jun. 2014), no. GT2014-26142, American Society of Mechanical Engineers.

[10] Gunn, E. J., Tooze, S. E., Hall, C. A., and Colin, Y., 2013. "An experimental study of loss sources in a fan operating with continuous inlet stagnation pressure distortion". Journal of Turbomachinery, 135(5), p. 051002.

[11] Gunn, E. J., and Hall, C. A., 2017. "Non-axisymmetric stator design for boundary layer ingesting fans". In Proceedings of the ASME Turbo Expo 2017 (Charlotte, North Carolina, USA, 26-30 Jun. 2017), no. GT2017-63082, American Society of Mechanical Engineers.

[12] Treaster, A. L., and Yocum, A. M., 1978. The calibration and application of five-hole probes. Tech. Rep. TM 78-19, Applied Research Laboratory, Jan.

[13] van Sluis, M., and Della Corte, B., 2020. Final PFC aircraft aerodynamic design and performance. CENTRELINE Deliverable 3.03, Delft University of Technology, Delft, Dec.

[14] Taylor, J. V., 2019. "Complete flow conditioning gauzes". Experiments in Fluids, 60(3), p. 35.

[15] Brandvik, T., and Pullan, G., 2011. "An accelerated 3d navier-stokes solver for flows in turbomachines". Journal of Turbomachinery, 133(2), p. 021025.

[16] Spalart, P. R., and Allmaras, S. R., 1994. "A one-equation turbulence model for aerodynamic flows". La Recherche Aérospatiale(1), pp. 5-21.

[17] NUMECA International, 2016, 2016. Autogrid5. Accessed July 13, 2018.

[18] Jerez Fidalgo, V., Hall, C. A., and Colin, Y., 2012. "A study of fan-distortion interaction within the nasa rotor 67 transonic stage". Journal of Turbomachinery, 134(5), p. 051011.

[19] Lieblein, S., Schwenk, F. C., and Broderick, R. L., 1953. Diffusion factor for estimating losses and limiting blade loadings in axial-flow-compressor blade elements. Research Memorandum RM-E53D01, National Advisory Committee for Aaeronautics, June. 


\section{LIST OF FIGURES}

1 Sketch of sources of distortion at the inlet of the fuselage fan. Adapted from Seitz et al. [4] . . . . . . . . . . . . . . . . . . . . . . 2

2 Meridional view of the BLI fan rig indicating locations of measurement stations $\ldots 4$

3 Flow conditioning gauzes, a) Clean, b) BLI distortion . . . . . . . . . . . . . . . . 6

4 Comparison of target, CFD and experimental measurements of axial velocity behind the conditioning gauzes at design point: a) Clean, b) BLI distortion . . . . . . . . . . 7

5 Meridional view of the BLI fan computational domain showing the fine (I) and coarse (II) mesh regions . . . . . . . . . . . . . . . . . . . . . 7

6 Computed spanwise distributions of: a) work loading and b) Lieblein's diffusion factor 9

$7 \quad$ Streamlines around the blade leading edge for fan $\mathrm{A}$ in BLI flow $\ldots \ldots$. . . . . . . 9

8 Rotor blade velocity triangle changes due to boundary layer ingestion for fan $A$ in BLl flow . . . . . . . . . . . . . . . . . . . . . 10

9 Pressure coefficient distributions of fan A and fan B in clean and BLI conditions - a) $10 \%$ span, b) $50 \%$ span, c) $90 \%$ span . . . . . . . . . . . . . . . . . . . . 10

10 Computed effect of distortion on fan A characteristics at constant rotor speed: a) stage loading, b) isentropic efficiency . . . . . . . . . . . . . . . . . . . 12

11 Computed rotor tip sections loss loops . . . . . . . . . . . . . . . . . . . 12

12 Rotor blade geometry: a) Metal angles, b) Solidity . . . . . . . . . . . . . . . . 13

13 Rotor blade sections at 10,50 and $90 \%$ span of fan $A$ and fan $B \ldots \ldots$

14 3D view of fans $A$ and $B$ rotor blades $\ldots \ldots \ldots \ldots \ldots$

15 Measured (symbols) and computed (lines) spanwise distributions of fan A - a) axial velocity, b) absolute swirl angle, c) stagnation pressure, c) radial angle . . . . . . . . 15

16 Measured (symbols) and computed (lines) spanwise distributions of fan B - a) axial velocity, b) stagnation pressure, c) absolute swirl angle, c) radial angle . . . . . . . 16

17 Measured spanwise distributions for fan A in clean and BLI flows- a) axial velocity, b) stagnation pressure, c) relative swirl angle, d) absolute swirl angle, e) specific angular momentum, f) radial angle . . . . . . . . . . . . . . . . . . 18 
18 Computed change in streamtube radius from station 1 relative to clean . . . . . . 19

19 Measured contours of stagnation pressure downstream of the OGV - a) fan A Clean, b) $\operatorname{fan} \mathrm{A}$ BLI, c) fan B BLI $\ldots \ldots \ldots \ldots \ldots$

20 Effect of distortion ingestion on the measured fan A characteristics at constant rotor speed: a) stage loading, b) isentropic efficiency . . . . . . . . . . . . . . . . . . . . 20

21 Measured spanwise distributions for fans A and B in BLI flow- a) axial velocity, b) stagnation pressure, c) relative swirl angle, d) absolute swirl angle, e) specific an-

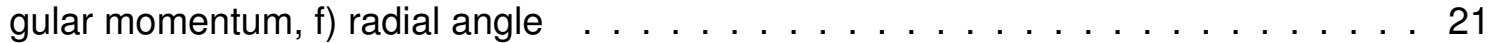

22 Comparison of measured fans $A$ and $B$ distorted characteristics at constant rotor speed. a) stage loading, b) isentropic efficiency . . . . . . . . . . . . . . . . . . . 22 
Journal of Turbomachinery

\section{LIST OF TABLES}

1 Design point parameters for the BLI fan rig $\ldots \ldots \ldots \ldots$

2 Experimental measurements of the aerodynamic performance of fan $A$ and $B$ at design point . . . . . . . . . . . . . . . . . . . . 20 